being traced within families by DNA analysis. Although the critical factor VIII gene is only about a tenth the size of the Duchenne's gene, it still is "very large," says Jane Gitschier of the Howard Hughes Medical Institute at the University of California (San Francisco). Recently, to improve such analysis, she and her colleagues adapted the polymerase chain reaction (PCR) procedure developed several years ago by researchers at Cetus Corp. (Emeryville, CA). Not only can tests now be conducted much more rapidly than before, but the gene amplification technique allows investigators to use much less material.

Huntington's disease is another inherited disorder whose onset can be predicted in some cases using DNAbased RFLP analysis. The key gene for this disease is carried near the tip of chromosome 4. Richard Myers of Boston University Medical Center, who is part of a Boston-area team focusing on Huntington's, says RFLP probes that are very closely linked to this site are now available for following inheritance patterns and predicting whether an individual will likely develop the disease. But he notes that it is also vital to analyze cell samples from other family members-preferably from across two generations-to track the illness accurately.

Diagnoses based on DNA analysis are not confined to hereditary diseases. In fact, methods using the polymerase chain reaction are being developed to diagnose infectious diseases including AIDS, hepatitis, and other virus-associated diseases, says John Sninsky of Cetus. In settings such as blood screening, he argues, a sensitive method using a "universal" probe to detect retroviruses could well prove valuable because the presence of any retrovirus might be reason enough to discard a blood sample. Also, use of amplification combined with sensitive detection methods could lead to the discovery of previously unrecognized viruses.

Some malignancies also lend themselves to DNA-based analysis because they are closely associated with chromosomal rearrangements, points out Mark Furth, director of diagnostics at Oncogene Science Inc. (Manhasset, NY). For example, a portion of chromosome 9 is moved onto chromosome 22 in malignant cells of individuals with chronic myelogenous leukemia. Although this event can be detected using traditional microscope-based cytogenetic analysis, he describes this procedure as "slow."

Furth explains, however, that because the chromosomal translocation includes the movement of the c-abl proto-oncogene, a more rapid alternative test is to detect altered RFLP patterns by DNA analysis using appropriate probes (see diagram). The procedure is sensitive enough to "pick up new junction fragments within peripheral blood cells," he says. Moreover, because RFLP analysis can detect low-frequency events, the technique may prove useful not only for confirmatory diagnosis but also for monitoring therapy. Oncogene Science's application for approval to use this diagnostic procedure is currently pending before the Food and Drug Administration.

In yet another twist, a DNA-based "fingerprint method" for analyzing forensic lab samples, determining family relations in paternity suits, and conducting diagnostic tests of genetic diseases has recently been offered as a commercial service by Cellmark Diagnostics (Germantown, MD). The molecular fingerprinting technology analyzes short, highly variable, but "stuttered" sequences of DNA that are scattered throughout an individual's genes. Because analysis depends on patterns throughout a genome, Cetus' PCR amplification cannot be readily applied to bail out investigators when they receive minuscule samples.

This medley of diagnostic developments promises to stir activity not only in the biotechnology industry and throughout the biomedical community, but also in the courts. Many perplexing legal and ethical issues must be faced, says Lori Andrews, an attorney associated with the American Bar Foundation (Chicago, IL). A specialist in genetics and the law, she raises the very practical question of how insurance companies will deal with screening test information about hereditary disorders. As new diagnostic technology becomes available, private insurers might pressure employers not to hire individuals with predispositions to costly ailments, she says, referring not only to Huntington's disease but also to diabetes, hypertension, and other health conditions with strong genetic determinants. Today, evidence of a preexisting condition is used in limited ways to disqualify affected individuals from insurance coverage. But Andrews points out that "with more sophisticated testing, virtually everything can become a 'preexisting condition,' even if an individual is asymptomatic." -Jeffrey L. Fox

\title{
FIRST CRYSTALIZATION FOR A LIGNINASE
}

\author{
IMAGE \\ UNAVAILABLE FOR \\ COPYRIGHT \\ REASONS
}

In this issue of Bio/Technology, Jan-Derk G. Smit, Armin Fietchter, and their colleagues at the Swiss Federal Institute of Technology (Zurich) and the Biozentrum (Basel) report the first crystalization of a ligninase. Lignin is the most abundant aromatic polymer in the world, but the details of its enzymatic degradation remained elusive until 1983. Since then, there has been an almost exponential rise in studies on the enzymology of lignin transformation, highlighted by two international symposia in Paris during the spring of 1987 . Crystals like the one shown, which is suitable for high-resolution X-ray analysis, provide the first step toward elucidating the three-dimensional structure of this important enzyme. 